\title{
Surface layer stiffness effects on fracture of polymer multilayers: a linear elastic model
}

\author{
P. S. Leevers ${ }^{*}$ \\ (Department of Mechanical Engineering, Imperial College London, London SW7 2AZ, UK; \\ p.leevers@imperial.ac.uk) \\ and L. Moreno \\ (University of Toronto, Department of Material Sciences and Engineering, \\ Toronto, M5S 3E4, Canada; moreno@mshri.on.ca)
}

\begin{abstract}
A tough thermoplastic polymer may show a transition to brittle behaviour when a skin of different properties forms on, or is painted or bonded onto, its free surfaces. A small-scale yielding, linear elastic analysis of the core material, in combination with an axisymmetric plate analysis of the skin, is used here to explore the role in this phenomenon of skin-core modulus inequality. When applied to the homogeneous (equal modulus) case, this very simple constraint model appears to provide independent support for the ASTM thickness criterion for plane-strain LEFM test validity. When applied to previously published impact fracture data from inhomogeneous (polyethylene-polypropylene) sandwich plates, the model successfully explains the shift in brittle-tough transition temperature precipitated by bonding a polypropylene skin to a polyethylene core. The model offers specific predictions for the effect, on transition temperature shift, of variables such as skin thickness and core properties; these predictions remain to be verified.
\end{abstract}

\section{Keywords}

Impact, fracture, polymers, constraint, surface layer 


\section{Introduction}

Many unreinforced thermoplastics develop much greater resistance to fracture under plane stress conditions than they do under plane strain conditions, i.e. where constraint around an internal crack front is high enough to suppress blunting flow. Such materials are generally put into service within the thickness/temperature regime where they benefit from this fact. Under such conditions they may show a transition to brittle behaviour [1-5] if a skin layer of different properties is sufficiently well bonded to the free surfaces to form a multilayer composite. This may be an issue for injection mouldings in which a core material is constrained between outer layers of a different material [2], or of the same material with different properties [6-9]. The effect of a skin layer on ductile-brittle transition has usually been attributed to decreased toughness [3,10], or to high crack speed in the surface layer [4].

In a previous paper [11] the authors investigated the fracture resistance of various three-layer composites, made by hot-laminating symmetrical stacks of polyolefin plates. Using a technique pioneered by Truss et al. [12] and extensively used more recently by Ingham [5], instrumented impact bend tests were carried out on Charpy specimens which had been sharply notched into the through-thickness face of the laminate. When tested individually within a conventional automotive service temperature range, all three of the polyolefins studied showed an impact fracture transition [11]. The temperature $T_{\mathrm{bt}}$ of this transition was pinpointed using load/displacement records from instrumented tests. All specimens tested near $T_{\text {bt }}$ exhibited plane-strain brittle rapid crack propagation (RCP), but below $T_{\mathrm{bt}} \mathrm{RCP}$ reached and broke the free surface opposite the notch, whilst above $T_{\mathrm{bt}} \mathrm{RCP}$ was arrested before it could do so. This brittle-tough transition may not be unique (in polyethylene, for example, there is another one related to long-term fracture), and it is certainly geometry dependent, but we have found $T_{\text {bt }}$ to be a useful index for materials and material systems subject to impact. The effect of bonding skin layers is usually to increase $T_{\mathrm{bt}}$ from its value for the core material alone.

Our objective here is to explain and, if possible, to predict quantitatively this increase in $T_{\mathrm{bt}}$. We proceed by assuming, as Ingham [5] and others concluded, that the effect of the skin is essentially similar to that of thickness-induced constraint. We use elastic plate theory in conjunction with classical linear elastic fracture mechanics to investigate the plane stress case in which the core dimples and the skin bends to remain compatible with it. Ingham ingeniously demonstrated the dominance of constraint by a skin over its toughness contribution by detaching the skin, before testing, and re-attaching it by staking with nylon pins; the 'embrittlement' effect was almost eliminated. Here the toughness of the skin is not considered at all; it is sufficient that the core material, like most polymers, exhibits a brittle-tough transition with increasing thickness. Particularly interesting results emerge when a simple model constructed on this basis is applied to the plane stress surface layer in a thick plate of homogenous material - the layered plane-stress plane-strain 'mixture' analysed variously by both Williams [13] and Ward [14].

\section{Analysis}

Consider a straight-through crack front in a plate of three-layer composite structure which is symmetrical around its mid-plane (Fig. 1a). A relatively thick core is clad with two well-bonded skins of a different material. The analysis will arrive at an estimate for the constraining effect of this skin on plastic deformation within a von Mises process zone. It is begun by calculating the core surface deflection (dimpling) which would occur around the crack under plane stress conditions [15] and in the absence of the skin. Plate theory $[16,17]$ is then applied to the skin in order to calculate the external pressure distribution needed to conform it to each deformed but stress free - surface. It is now assumed that this 'pressure' distribution is actually applied to the skin by tensile tractions acting through the skin-core interface. Comparing the magnitude of these tensile tractions to that of the through-thickness tensile stresses which would be generated by plane strain constraint provides, finally, a criterion for skin-induced fracture mode transition. 
Figure $1 \mathrm{~b}$ represents a cross-section in the plane of a flat, through-thickness, straight-fronted crack in a flat plate of total thickness $B$. This plate consists of a core, $B_{1}$ thick, and two identical surface skins of thickness $s$; the crack has arrived from the left and $r$ is measured forwards on its line of extension, i.e. at $\theta=0$.

Within the core, the through-thickness strain under plane stress conditions $\left(\sigma_{z z}=0\right)$ is $[13,18]$

$$
e_{z z}=\frac{-v_{1}}{E_{1}}\left(\sigma_{r r}+\sigma_{\theta \theta}\right)
$$

where $E_{1}$ and $v_{1}$ are tensile modulus and Poisson's ratio, and subscript 1 identifies the core material. The through-thickness normal stress under plane strain conditions $\left(e_{z z}=0\right)$ is

$$
\sigma_{z z}=v_{1}\left(\sigma_{r r}+\sigma_{\theta \theta}\right)
$$

Taking into account only the singular part of the near-tip stress field:

$$
\sigma_{r r}+\sigma_{\theta \theta}=2 \frac{K_{\mathrm{I}}}{\sqrt{2 \pi r}} \cos \left(\frac{1}{2} \theta\right)
$$

so that if the core is under plane stress conditions each of its surfaces will dimple by a displacement

$$
w=-\frac{1}{2} B_{1} e_{z z}=v_{1} \frac{B_{1}}{E_{1}} \frac{K_{\mathrm{I}}}{\sqrt{2 \pi}} \cos \left(\frac{1}{2} \theta\right) r^{-1 / 2} .
$$

As the radius $r$ from the crack tip tends to zero, this displacement shows the expected singularity. As usual, the ' $K$ field' can represent stresses and strains only:

(a) within a radius small enough for $K$ dominance, and

(b) outside a radius within which stresses are limited by plastic flow.

Figure 1(c) shows a sketch of the singular dimpling displacement (dashed line) and of how near-tip plastic flow might mitigate it.

The displacement profile is locally axisymmetric around the line of crack extension $\theta=0$. We can therefore use axisymmetric plate theory [16] to estimate the tensile interface traction distribution $\sigma_{\mathrm{i}}(r)$ which would be needed to draw a surface skin of thickness $s$ down onto the deformed core surface (Fig. 1d):

$$
\sigma_{\mathrm{i}}=\frac{E_{2} s^{3}}{6\left(1-v_{2}^{2}\right)} \frac{1}{r} \frac{\mathrm{d}}{\mathrm{d} r}\left\{r \frac{\mathrm{d}}{\mathrm{d} r}\left[\frac{1}{r} \frac{\mathrm{d}}{\mathrm{d} r}\left(r \frac{\mathrm{d} w}{\mathrm{~d} r}\right)\right]\right\}
$$

where subscript 2 identifies skin properties. Hence from (4) and (5):

$$
\sigma_{\mathrm{i}}=\frac{1}{12 \sqrt{2 \pi}} \frac{v_{1}}{\left(1-v_{2}^{2}\right)} \frac{E_{2}}{E_{1}} B_{1} s^{3} K_{\mathrm{I}} \frac{1}{r} \frac{\mathrm{d}}{\mathrm{d} r}\left\{r \frac{\mathrm{d}}{\mathrm{d} r}\left[\frac{1}{r} \frac{\mathrm{d}}{\mathrm{d} r}\left(r \frac{\mathrm{d}}{\mathrm{d} r}\left(r^{-1 / 2}\right)\right)\right]\right\} .
$$

Differentiating repeatedly we arrive at

$$
\frac{1}{r} \frac{\mathrm{d}}{\mathrm{d} r}\left\{r \frac{\mathrm{d}}{\mathrm{d} r}\left[\frac{1}{r} \frac{\mathrm{d}}{\mathrm{d} r}\left(r\left(r^{-1 / 2}\right)\right)\right]\right\}=\frac{25}{16} r^{-9 / 2}
$$

so that

$$
\sigma_{\mathrm{i}}=\frac{25}{192 \sqrt{2 \pi}} \frac{v_{1}}{\left(1-v_{2}^{2}\right)} \frac{E_{2}}{E_{1}} B_{1} s^{3} K_{\mathrm{I}} r^{-9 / 2} .
$$

Of course, if tensile tractions did in fact act between the skin and the core surface, the core surface would no longer be stress free and the deflection would decrease. The calculated interface stress must therefore represent an upper bound.

This estimate of tensile interface stress is compared to the through-thickness tensile stress which would be generated within the core under fully plane strain conditions, induced by constraint from the core material alone. From Eqns (2) and (3) this is 


$$
\sigma_{z z}=2 v_{1} \frac{K_{\mathrm{I}}}{\sqrt{2 \pi r}}
$$

at $\theta=0$. The $K$-dominance conditions mentioned above must also be borne in mind for both Eqns. (8) and (9), neither of which represents an equilibrated stress field. Nevertheless, we can estimate the significance of the interface stress given by (8) in comparison with the throughthickness stress generated by plane-strain conditions, by examining the dimensionless ratio

$$
C \equiv \frac{\sigma_{\mathrm{i}}}{\sigma_{z z}}=\frac{25}{384} \frac{1}{\left(1-v_{2}^{2}\right)} \frac{E_{2}}{E_{1}} B_{1} s^{3} r^{-4} \text {. }
$$

Setting $C=1$ we find that, at a specific distance $r$ ahead of a crack tip, plane strain conditions could be induced by the skin into a plate previously under plane stress conditions if

$$
\frac{25}{384} \frac{1}{\left(1-v_{2}^{2}\right)} \frac{E_{2}}{E_{1}} \frac{B_{1}}{r}\left(\frac{s}{r}\right)^{3}>1 \text {. }
$$

Now, under the plane stress conditions which pertained before the skin applied these throughthickness tractions the radius of a von Mises plastic zone at $\theta=0$ was

$$
r_{\mathrm{p} 1}=\frac{1}{2 \pi} \frac{E_{1} G}{\sigma_{\mathrm{c}}^{2}} .
$$

In order to estimate whether the skin would have a significant effect on the development of such a zone we test condition (11) at the plane-stress plastic zone size $r=r_{\mathrm{p}}$, since it is at this point that the constraint level can be expected to determine the fracture mode. Remembering that we considered a plate whose structure is symmetrical around its centre plane, so that $B=B_{1}+2 s$, Eqn (11) finally becomes

$$
\frac{25}{384} \frac{1}{\left(1-v_{2}^{2}\right)} \frac{E_{2}}{E_{1}}\left(\frac{B}{r_{\mathrm{p}}}-2 \frac{s}{r_{\mathrm{p}}}\right)\left(\frac{s}{r_{\mathrm{p}}}\right)^{3}>1 .
$$

\subsection{Homogeneous material represented as a layered structure}

Consider first a flat plate of a single-material, i.e. one whose 'core' and 'skin' materials have the same modulus $E_{1}=E_{2}$. The layer interface position is defined by the difference in stress states, at the core plastic zone radius $r_{\mathrm{p} 1}$, between the constraining skin (under plane stress) and the constrained core (under plane strain). Thus $s$ represents the expected thickness of a plane stress surface layer. In polymers this layer is often distinguished by stress whitening, and is usually tougher than the core.

For this case, re-arranging Eqn. (13) with $B / r_{\mathrm{p}}$ as subject gives

$$
\frac{B}{r_{\mathrm{p}}}=\frac{384}{25}\left(1-v^{2}\right)\left(\frac{s}{r_{\mathrm{p}}}\right)^{-3}+2 \frac{s}{r_{\mathrm{p}}}
$$

Equation (14) has been solved explicitly for an appropriate range of $s / r_{\mathrm{p}}$, and plotted in Fig. 2 as $s / r_{\mathrm{p}}$ versus $B / r_{\mathrm{p}}$ for $v=0.35$. The inverse function $B / r_{\mathrm{p}}$ versus $s / r_{\mathrm{p}}$ has a minimum, at

$$
\frac{s}{r_{\mathrm{p}}}=2 \sqrt{\frac{6}{5}}\left(1-v^{2}\right)^{1 / 4}=2.1909\left(1-v^{2}\right)^{1 / 4}
$$

i.e., from Eqn. (14), at

$$
\frac{B}{r_{\mathrm{p}}}=4\left[\sqrt{\frac{2}{15}}+\sqrt{\frac{6}{5}}\right\rfloor\left(1-v^{2}\right)^{1 / 4}=5.842\left(1-v^{2}\right)^{1 / 4},
$$


which is virtually independent of Poisson's ratio. Eliminating $r_{p}$ between these two equations shows that the minimum, which appears as a fold back on the left hand side of Fig. 2, occurs exactly and unconditionally at $s=\frac{3}{8} B$. For $B / r_{\mathrm{p}}$ values exceeding the critical value given by Eqn (15), $s / r_{\mathrm{p}}$ versus $B / r_{\mathrm{p}}$ therefore has two branches; the upper one (seen curving away to the right at the top of Fig. 2) represents solutions in which a thicker surface region maintains the same stress in a core which is much thinner and which, therefore, dimples much less under the same stress intensity. For plate thicknesses below the critical value, there is no room for either layer configuration to generate plane strain. Thus, according to this analysis, a crack front in a plate thinner than $5.842\left(1-v^{2}\right)^{1 / 4} r_{\mathrm{p}}$ is unconditionally in pure plane stress.

Now, for thicker plates, recall the ASTM thickness criterion for the validity of a plane strain fracture test:

$$
B>2.5\left(\frac{E G_{\mathrm{c}}}{\sigma_{\mathrm{y}}^{2}}\right)=5 \pi r_{\mathrm{p}} .
$$

Figure 2 highlights a striking result: the ASTM plane-strain criterion (which has a long history of empirical verification) corresponds in the present analysis to a plane stress layer almost exactly $r_{\mathrm{p}}$ thick. Such a plastic zone can be visualised (e.g. [14]) as being bounded by a conical surface of $45^{\circ}$ slip planes. This may well be a good representation for many materials whose fracture behaviour underpins the ASTM criterion, but it is not so for many others. Because $s / r_{\mathrm{p}}$ declines gently with increasing $B / r_{\mathrm{p}}$, derivation of the ASTM criterion from the plate model might seem rather ill-conditioned, but simply substituting $s=r_{\mathrm{p}}$ into Eqn. (14) provides the results shown in Fig. 3 which do not depart significantly from Eqn. (16) for any realistic value of Poisson's ratio. Also shown on Fig. 3 is the plane stress criterion of Eqn. (15) which, as shown, can simply be expressed as:

$$
B<0.9\left(\frac{E G_{\mathrm{c}}}{\sigma_{\mathrm{y}}^{2}}\right)=1.8 \pi r_{\mathrm{p}} .
$$

For a plate whose thickness exceeds the ASTM validity criterion, the present analysis predicts a further decline in plane stress layer thickness. However, Saint-Venant's principle forbids surface dimpling from increasing without limit with increasing thickness. A plate thicker than $5 \pi r_{\mathrm{p}}$ can conveniently be modelled as a multi-layered structure, consisting of:

(a) a plane strain core of infinite through-thickness rigidity;

(b) a region about $(2.5 \pi-1) r_{\mathrm{p} 1}$ thick which deforms in plane stress but whose process zone boundary is effectively constrained to plane strain; and

(c) a surface region $r_{\mathrm{p}}$ thick whose process zone is under plane stress.

This model is illustrated in Figs. 2 by replacing the solution of Eqn. (14) by $s / r_{\mathrm{p}}=1$ for $B / r_{\mathrm{p}} \geq 5 \pi$.

Having established consistency between the present model and the ASTM plane strain validity criterion, we return to the subject of layered structures.

\subsection{Inhomogeneous multilayer structures}

For a core with well-bonded skins of modulus $E_{2}$, Eqn. (13) gives a plane strain condition

$$
\frac{B}{r_{\mathrm{p} 1}}>\frac{384}{25} \frac{\left(1-v_{2}^{2}\right)}{E_{2} / E_{1}}\left(\frac{s}{r_{\mathrm{p} 1}}\right)^{-3}+2 \frac{s}{r_{\mathrm{p} 1}}
$$

where $r_{\mathrm{p} 1}$ is the plastic zone radius for the core material. The skin is now essentially distinct from the core, if only in modulus, so that $s$ is a predetermined parameter. Equation (18) generalises Eqn. (14); it constitutes a criterion for fully plane strain conditions which is fully consistent with the ASTM criterion for a homogeneous material. Figure 4 plots the $s$ versus $B$ 
locus for a number of $E_{2} / E_{1}$ ratios, including unity. Again, solutions lying on the branch below the minimum

$$
\frac{B}{r_{\mathrm{p} 1}}=5.842\left(\frac{1-v_{2}^{2}}{E_{2} / E_{1}}\right)^{1 / 4}
$$

are excluded and replaced by a vertical line from the critical point to the full-thickness skin condition $s=\frac{1}{2} B$. To discount any contribution to dimpling from core material lying within the 'through thickness rigid' region, solutions are also restricted by the condition

$$
\frac{B}{r_{\mathrm{p} 1}}-2 \frac{s}{r_{\mathrm{p} 1}} \leq(5 \pi-2) \text {. }
$$

After these corrections, the region above and to the right of each curve is that for which the transition to plane strain conditions is complete.

\subsection{Transition temperature shifts}

The problem referred to in the introduction was that of polymer sandwich structures having a higher transition temperature than that of solid core. Figure 5 re-plots the computed results of Fig. 4 in a form which can be applied more directly where the value of $s / B$ is predetermined. A change in temperature intervenes through the parameters $r_{p 1}$ - which usually increases gently with temperature, so that $B / r_{\mathrm{p} 1}$ decreases - and $E_{2} / E_{1}$, which may increase or decrease depending on the materials chosen. The temperature dependence of these parameters must first be determined to scale the dimensionless variables into usable results.

The use of this analysis can now be explored for a specific system, studied in a previous paper [11]. This consisted of a high-density $\left(958 \mathrm{~kg} \mathrm{~m}^{-3}\right)$, selectively branched, low-flow PE, and an equally low flow PP copolymer of density $905 \mathrm{~kg} \mathrm{~m}^{-3}$ (no further details of these experimental grades were available). A compression moulded $1 \mathrm{~mm}$ PP copolymer skin was hot-bonded to each side of a thicker compression moulded PE slab to produce a sandwich structure $12 \mathrm{~mm}$ thick. Coupons $(B=10 \mathrm{~mm}) \times(W=10 \mathrm{~mm}) \times 75 \mathrm{~mm}$ length were cut from this plate and tested under impact bend loading at $1 \mathrm{~m} \mathrm{~s}^{-1}$ using the ISO 17281 fracture-mechanics methodology. A single initial notch depth of $0.25 \mathrm{~W}$ was used; the notch was oriented so that the crack front advanced normal to the sandwich plate surface as assumed in this analysis. The 'arrest transition' temperature $T_{\mathrm{bt}}$ was defined as that above which the rapid crack jump arrested before breaking the free surface opposite the notch.

ISO 17281 tests on full-thickness monolithic core material $(\mathrm{PE})$ plate generated $G_{\mathrm{c}}$ values which could be expressed using

$$
G_{\mathrm{c}}\left(\mathrm{kJ} \mathrm{m}^{-2}\right)=10.2+0.0185 T\left({ }^{\circ} \mathrm{C}\right)
$$

and $T_{\mathrm{bt}}$ was measured to be $-11^{\circ} \mathrm{C}$. Monolithic skin material tested at the same thickness gave a transition temperature of $+11^{\circ} \mathrm{C}$. The bonded PP surface layers caused a decisive increase in $T_{\text {bt }}$ from $-11^{\circ} \mathrm{C}$ to $+10^{\circ} \mathrm{C}$. Using the Reversed Charpy method [19], an appropriately constrained yield stress was determined as a function of temperature $T$ and the results could be represented as

$$
\sigma_{\mathrm{y}}=52.6(\mathrm{MPa})-0.392 T\left({ }^{\circ} \mathrm{C}\right) .
$$

Modulus vs. temperature was determined for both core and skin materials, using both DMTA (Fig. 6) and a high-speed (1 m/s) variant of the standard flexural test. From these results Figure 7 plots both core (PE) and skin (PP copolymer) material plastic zone size. Notice that $r_{\mathrm{p} 1}$ for the $\mathrm{PE}$ core material increases only at a modest rate with temperature $T$ before rolling over a maximum towards room temperature; there is little here to hint at any transition. For the PP copolymer skin material, on the other hand, the plastic zone size maximum is even more pronounced, due to rapid softening above the glass transition temperature at about $10^{\circ} \mathrm{C}$. To explain data from various thicknesses of epoxy skin on a polycarbonate core, Moore [20] proposed that brittle behaviour would be expected when the skin thickness exceeded its own

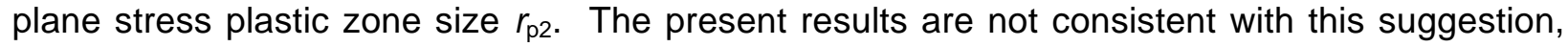


since the plastic zone size never attains the skin thickness and does not show monotonic dependence on temperature.

To test the present hypothesis that the transition is an effect of skin constraint on core yielding, Fig. 8 uses the $r_{\mathrm{p} 1}$ results to plot the constraint factor

$$
C \equiv \frac{25}{384} \frac{1}{\left(1-v_{2}^{2}\right)} \frac{E_{2}}{E_{1}}\left(\frac{B}{r_{\mathrm{p}}}-2 \frac{s}{r_{\mathrm{p}}}\right)\left(\frac{s}{r_{\mathrm{p}}}\right)^{3}
$$

as a function of temperature. This passes through the chosen reference value of unity at $8.3^{\circ} \mathrm{C}$, so that according to the ASTM criterion a transition to plane strain is effectively complete.

\section{Discussion}

The plane-strain transition temperature predicted using this analysis, $8.3^{\circ} \mathrm{C}$, agrees encouragingly well with the measured transition temperature of $+10^{\circ} \mathrm{C}$. It is interesting to compare two other significant temperatures: that at which the core plastic zone size becomes equal to $1 \mathrm{~mm}\left(16^{\circ} \mathrm{C}\right)$ and that at which the core and skin moduli become equal $\left(9.1^{\circ} \mathrm{C}\right)$.

How can the impact fracture arrest transition relate to the ASTM 'full-plane strain' criterion? It is important to recognise that despite the physical basis of the present model, it is implemented through a 'characteristic value' criterion $(C=1)$ which is not directly related to impact RCP arrest. An empirical relationship between a 'full plane strain' criterion and impact RCP arrest could be determined for a single material, but this would involve precisely locating the skin/core (i.e. plane-stress/plane-strain) interface in a monolithic material, and this is difficult to do. It is therefore not possible using the present model to predict a transition temperature for the monolithic material, although the elements of a model which could do so have been demonstrated [21].

This lack of a reference state is also true of the ASTM criterion; it could hardly matter very much whether test conditions satisfied the criterion or not if the plane stress and plane strain toughnesses were comparable. If the single result presented here proves to represent a wider correspondence between the model and reality, then it can be concluded that principal source of surface embrittlement is skin/core modulus inequality. However, it might well still be possible for a skin to compensate, by its high toughness, for the core properties it has disabled by constraint from its high stiffness. Ingham [5] found this to be the case for a methacrylate butadiene styrene toughened PVC bonded to a PVC core.

For this particular polyethylene-polypropylene system, Figs. 9 and 10 illustrate two sensitivity issues. In Figure 9 the principal property of the core material - its characteristic plane stress plastic zone size $r_{\mathrm{p} 1}$ - is varied from one-half to twice its value at the predicted transition temperature of $8.3^{\circ} \mathrm{C}$. Since the effect of modulus is best considered with reference to that of the skin, this is equivalent to increasing core impact fracture resistance from $0.5 G_{c}$ to $2 G_{c}$ or decreasing core yield stress from $2^{1 / 2} \sigma_{\mathrm{y}}$ to $2^{-1 / 2} \sigma_{\mathrm{y}}$ (see Eqn. (12)). Note that increasing impact rate in thermoplastics normally increases $\sigma_{y}$ and decreases $G_{c}$. Clearly in each case the result is a substantial shift in transition temperature, because the boundary of a larger plane stress zone is less susceptible to surface constraint by a given skin. It is not necessarily impossible for the multilayer transition temperature to be below that measured for the skin or above that measured for the core. Both of these values were measured on material of the same thickness as the multilayer, and will be thickness sensitive.

Figure 10 considers the effect of a change in skin thickness s. Both Verpy et al. [1] and Ramsteiner [3] noted that thick coatings of hard material had a more deleterious effect on multilayer ductility than did thin ones. Although Ramsteiner suggested that this was because the composite surface notch created by coating fracture was larger, he was also aware of the importance of coating constraint. Eqn. (5) shows that the constraint effect depends on the third power of $s$, and therefore has predominant significance. It should be mentioned that the compression moulded skin sheets used in these experiments varied somewhat in thickness, 
and this sensitivity will have contributed to scatter. Many aspects of this model remain to be explored in future work, and close attention to the precision and variance of skin thickness will be essential.

\section{Conclusions}

The fracture properties of thermoplastic polymers are sometimes negatively influenced by the presence of a well-bonded surface layer with different properties. The present analysis focuses purely on the part played in this phenomenon by elastic constraint from the skin material on near-tip processes in the core material. The skin is modelled as an axisymmetrical elastic plate and the core as a two-dimensional elastic continuum with small-scale yielding. The resulting model is compatible with the thickness requirement for ASTM validity criterion for fully plane strain conditions in a uniform (single material) plate, if the plane stress 'skin' is assumed to be one plastic zone size in thickness; it also leads us to expect fully plane stress conditions for a sheet whose thickness is less than 0.36 times the ASTM plane strain minimum. The model generalises these criteria to multilayer structures, and satisfactorily explains the impact fracture transition temperature shift in a polypropylene copolymer skinned polyethylene structure. Further predictions remain to be verified, and the role of skin toughness remains to be explored.

\section{References}

1. Verpy C. Gacougnolle JL. Dragon A. Vanlerberghe A. Chesneau A. Cozette F. The surface embrittlement of a ductile blend due to a brittle paint layer. Progress in Organic Coatings. 1994;24:115-129.

2. Karger-Kosis J. Csikai I. Skin-core morphology and failure of injection-molded specimens of impact-modified polypropylene blends. Polymer Engineering and Science. 1987;27(4): 241-253.

3. Ramsteiner F. Jaworek T. Weber M. Forster S. Scratch resistance and embrittlement of coated polymers. Polymer Testing. 2003;22:439-451.

4. Könczöl L. Döll W. Edinger B. On the mechanisms of surface embrittlement of ductile thermoplastics by brittle surface layers. In: Proceedings of the 8th International Conference on Deformation, Yield and Fracture of Polymers. London: IoM Communications, 1991.

5. Ingham EJ. The development of impact toughness and resistance to slow crack growth in modified polyvinyl chloride and polyethylene pipe grade polymers. PhD Thesis, Manchester Metropolitan University, UK, 2003.

6. So P. Broutman LJ. The effect of surface embrittlement on the mechanical behaviour of rubber modified polymers. Polymer Engineering and Science. 1982;22:888-894.

7. So P. Broutman LJ. The fracture behaviour of surface embrittled polymers. Polymer Engineering and Science. 1986;26:1173-1179.

8. Dear JP. Surface effects on the fracture of polymers. Materials Science and Engineering. 1991;A147:257-265.

9. Fayolle B. Audovin L. Verdu J. Oxidation induced embrittlement in polymers - a tensile testing study. Polymer Degradation and Stability. 2000;70:333-340.

10. Priebe E. Stabenov J. Aging effects in high impact SAN copolymers - relationship between surface damage and impact strength of ABS. Kunststoffe. 1974;64:9.

11. Moreno L. Leevers PS. Impact fracture toughness of polyethylene/polypropylene multilayers. Polymer Engineering and Science. In press.

12. Truss RW. Duckett RA. Ward IM. Polym. Eng. Sci. 1983;23:708.

13. Williams JG. Fracture Mechanics of Polymers. Chichester: Ellis Horwood, 1984.

14. Ward IM, Hadley DW. Mechanical Properties of Solid Polymers. Chichester: John Wiley, 1993.

15. Tetelman AS. McEvily AJ Jr. Fracture of Structural Materials. New York: John Wiley, 1967.

16. Timoshenko S. Theory of Plates and Shells. New York: McGraw-Hill, 1959.

17. Reddy J N. Theory and Analysis of Elastic Plates. London: Taylor and Francis, 1999.

18. Kanninen MF. Popelar C H. Advanced Fracture Mechanics. Oxford: Oxford Engineering Science, 1985. 
19. Davies P. Ritchie SJ. Leevers PS. Brittle-tough transition of rapid crack propagation in polyethylene. Polymer. 1998;39(25):6657-6663.

20. Moore, D R. Prediction of ductile-brittle transitions in coating materials. In: Williams JG, Moore DR editors. Application of Fracture Mechanics to Polymers, Adhesives and Composites. Amsterdam: Elsevier, 2003 (in press).

21 Leevers PS. Hazra S. Hillmansen S. Impact resistance of thermoplastics: prediction from bulk properties. Plastics Rubbers and Composites Processing and Applications. 2000;29(9): 460-467.

\section{List of Figures}

1 (a) Unloaded through-thickness crack front in a multilayer plate; (b) cross-section of crack plane near front; (c) plane stress core deformation on loading of crack front; (d) axisymmetric bending to restore skin-core contact.

2 Core-skin model for a homogeneous material: plane stress layer thickness as a function of total plate thickness, both normalised against plastic zone size $r_{\mathrm{p}}$

3 Core-skin model criteria, as functions of Poisson's ratio, for 'pure plane stress' and 'pure plane strain' plate thickness

$4 \quad$ Plane strain criteria for symmetrical multilayers of two materials differing in modulus

5 Results of Fig. 4 renormalised against core plastic zone size

$6 \quad$ Skin and core modulus for the polymer multilayer structure, measured using DMTA

$7 \quad$ Plane stress plastic zone size of polymer multilayer skin and core materials

8 Skin-core constraint factor as a function of temperature for a polymer multilayer, showing core and skin transition temperatures at the multilayer thickness

9 Variation of predicted multilayer transition temperature with skin thickness

10 Variation of predicted multilayer transition temperature with core plastic zone size 
(a) multilayer thickness

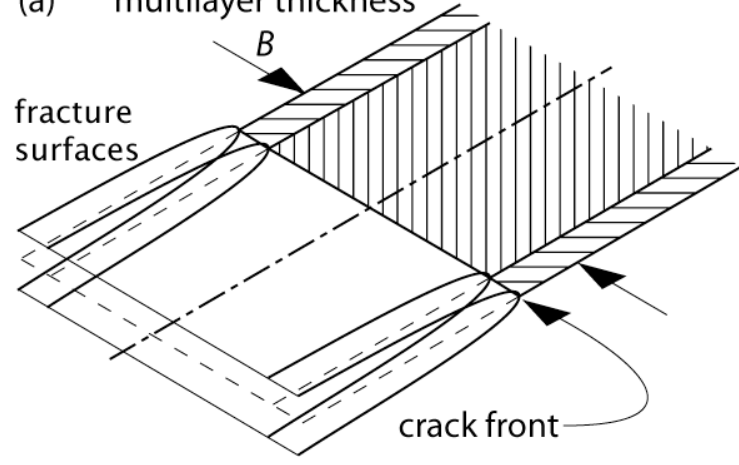

(c)

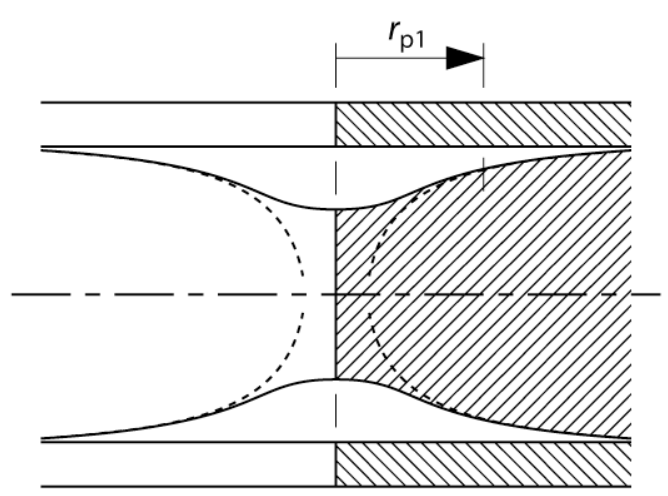

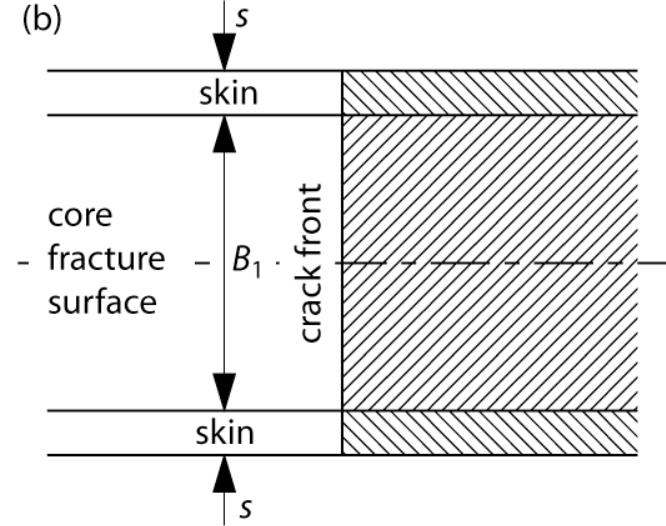

(d)

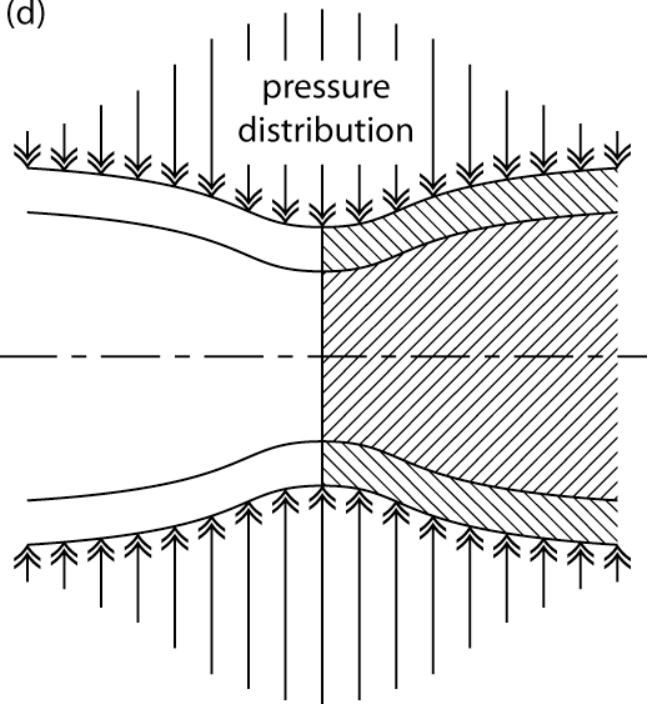




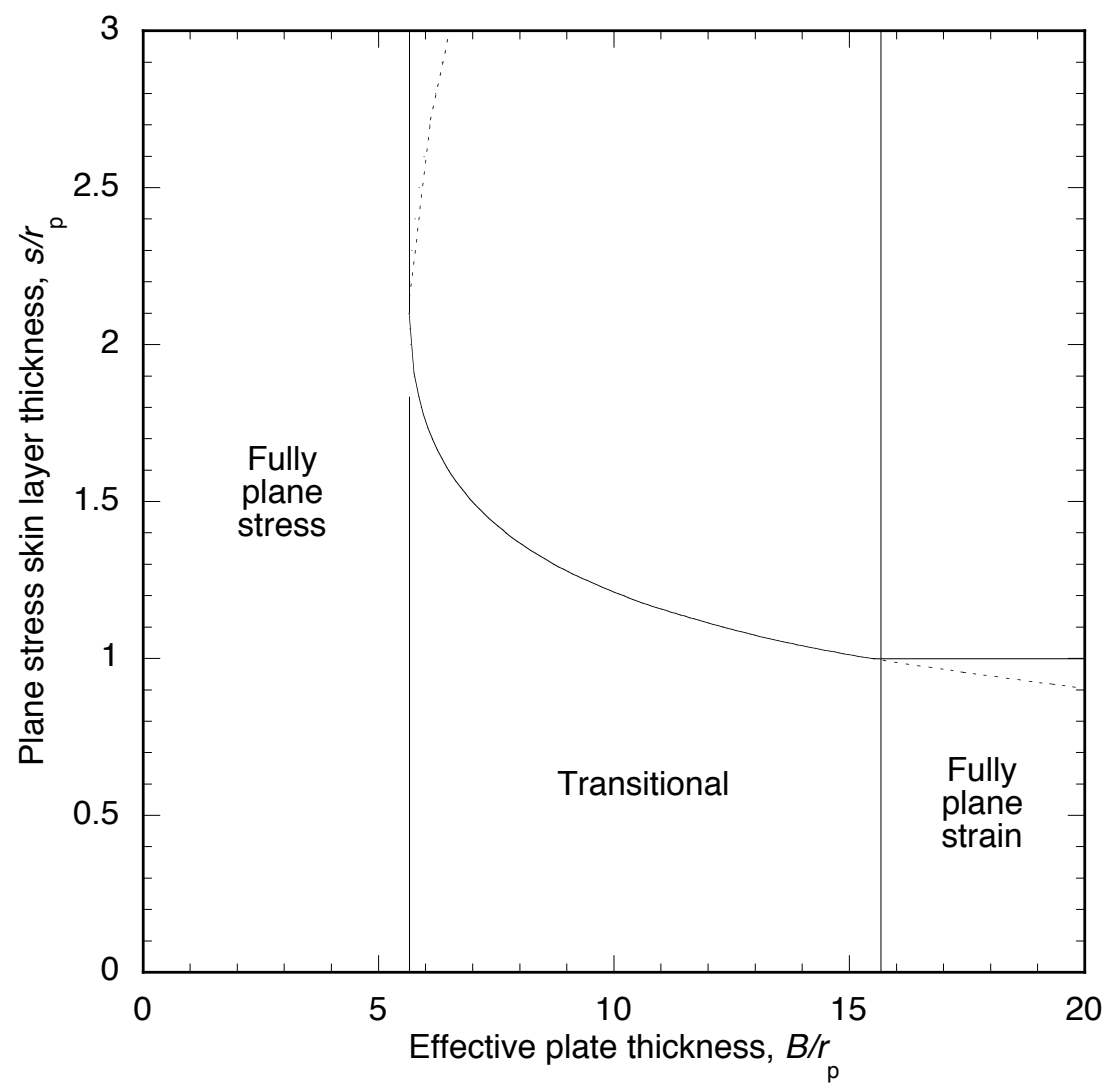




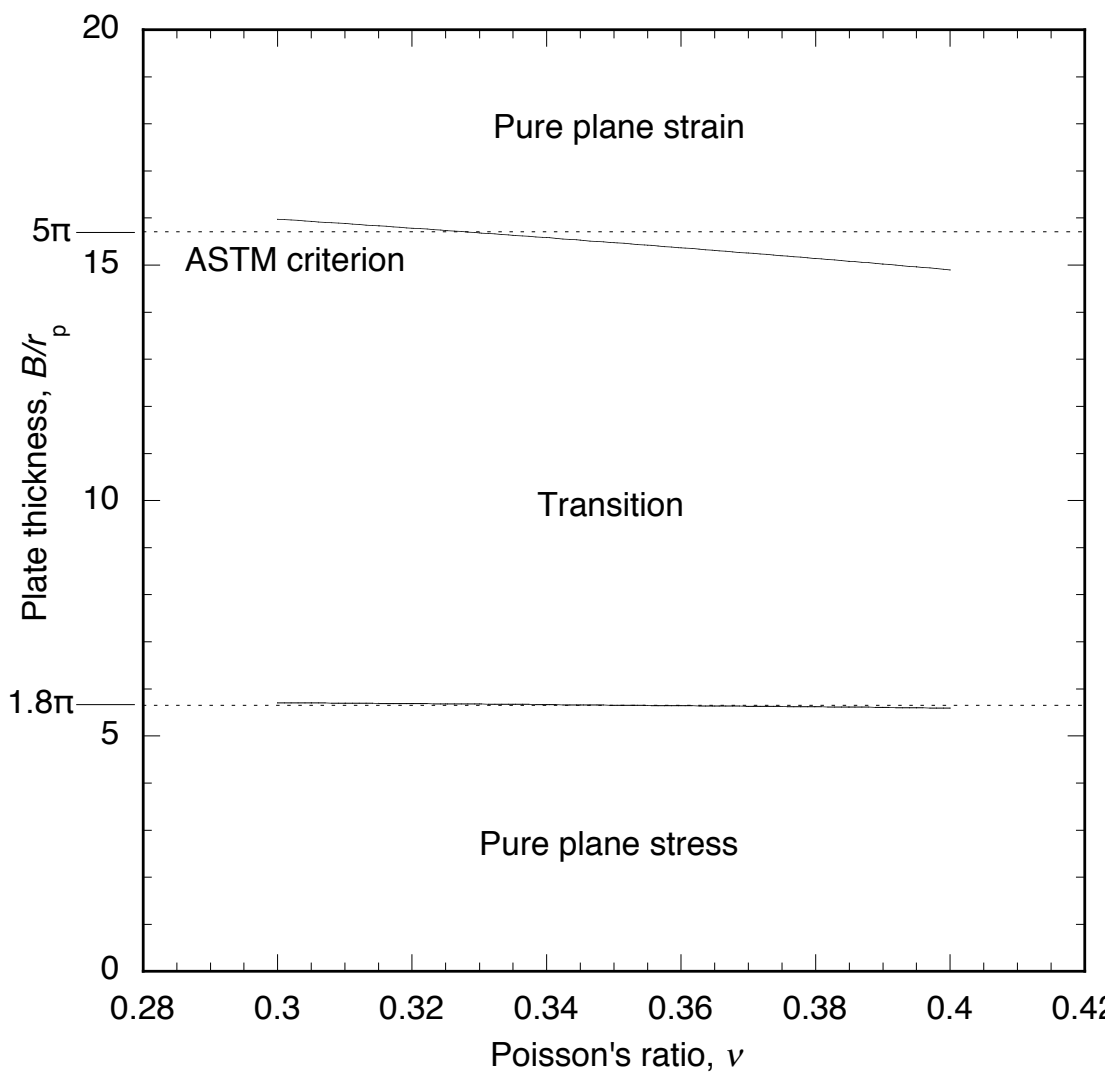




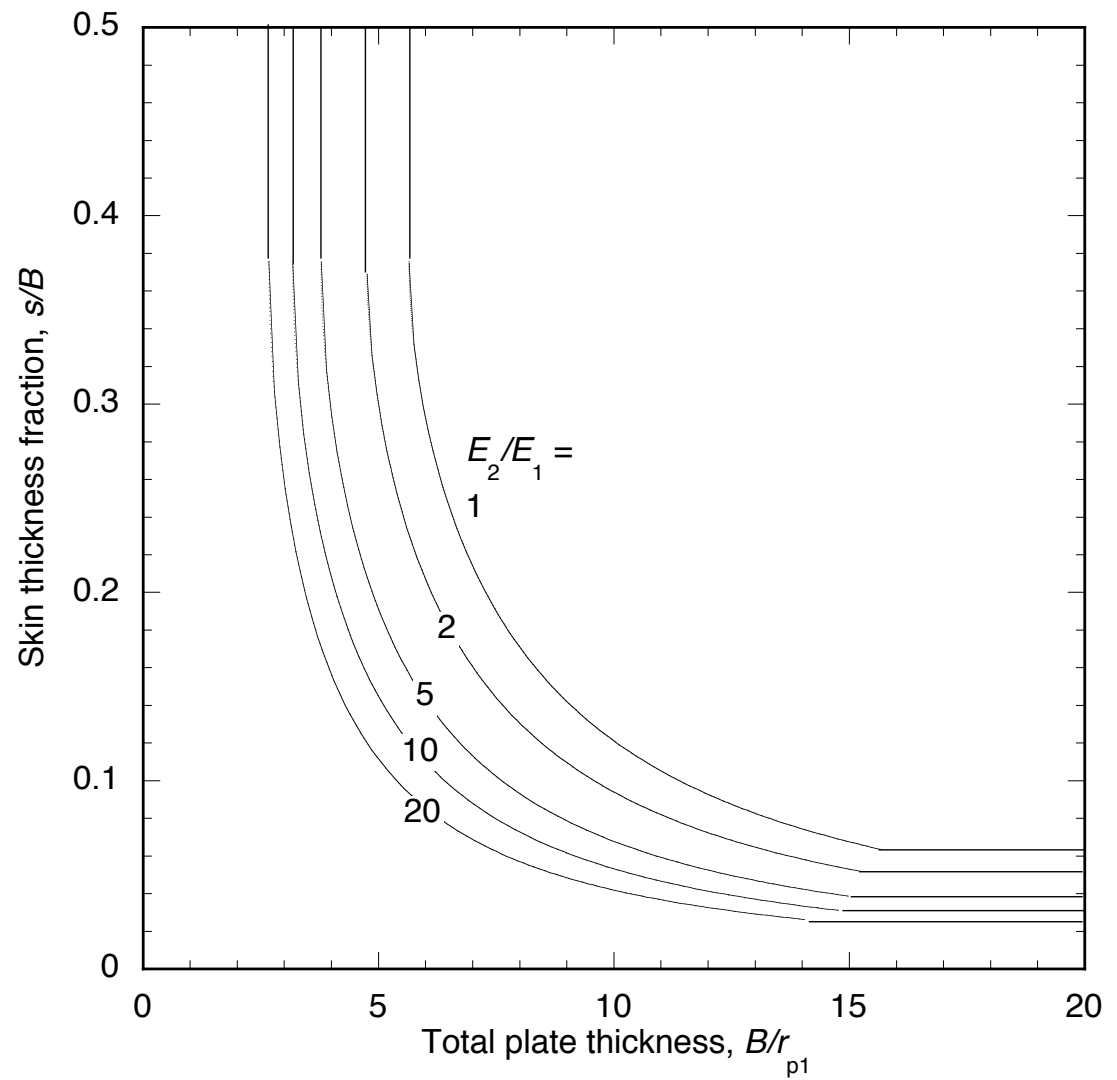




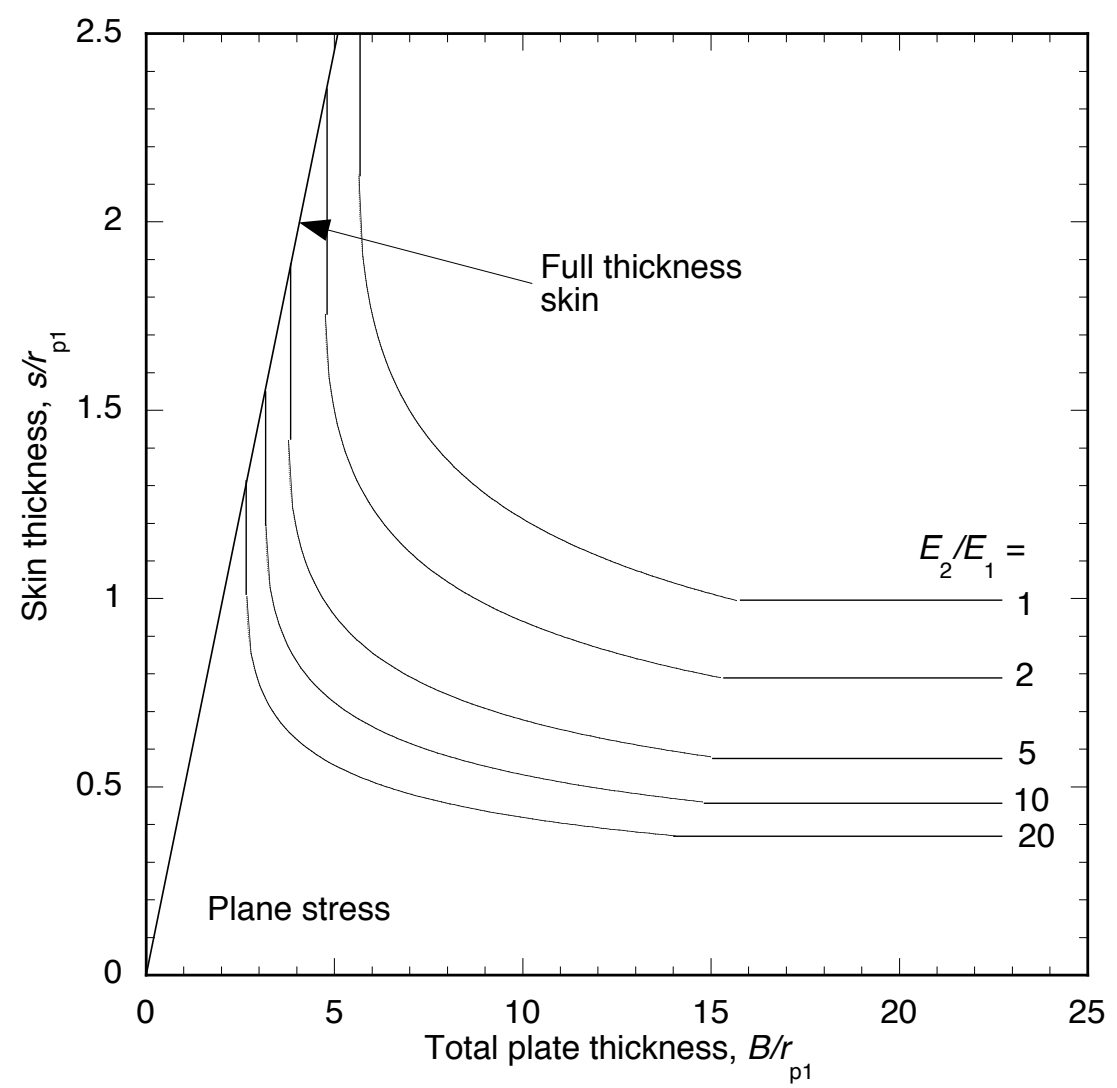




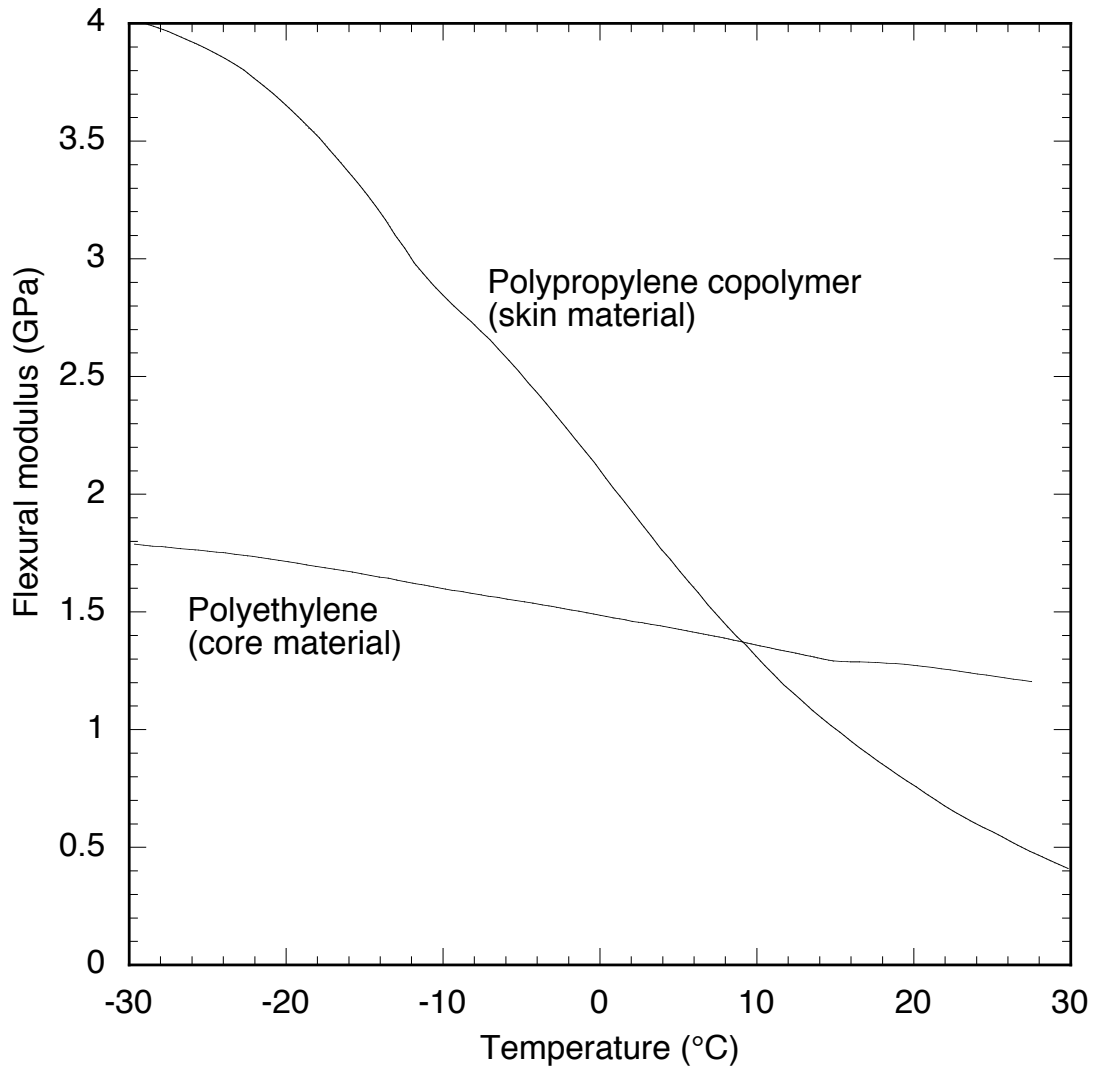




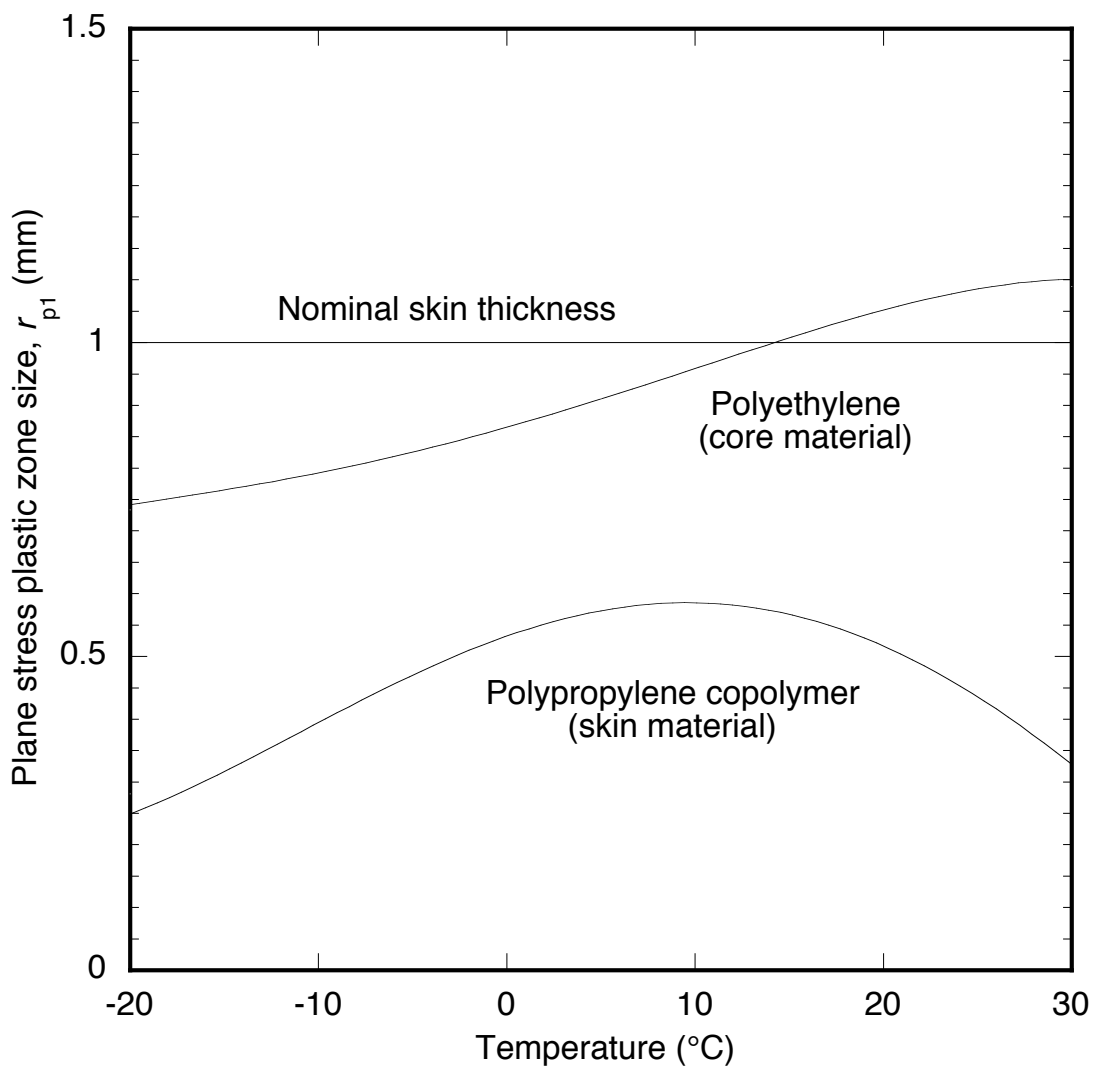




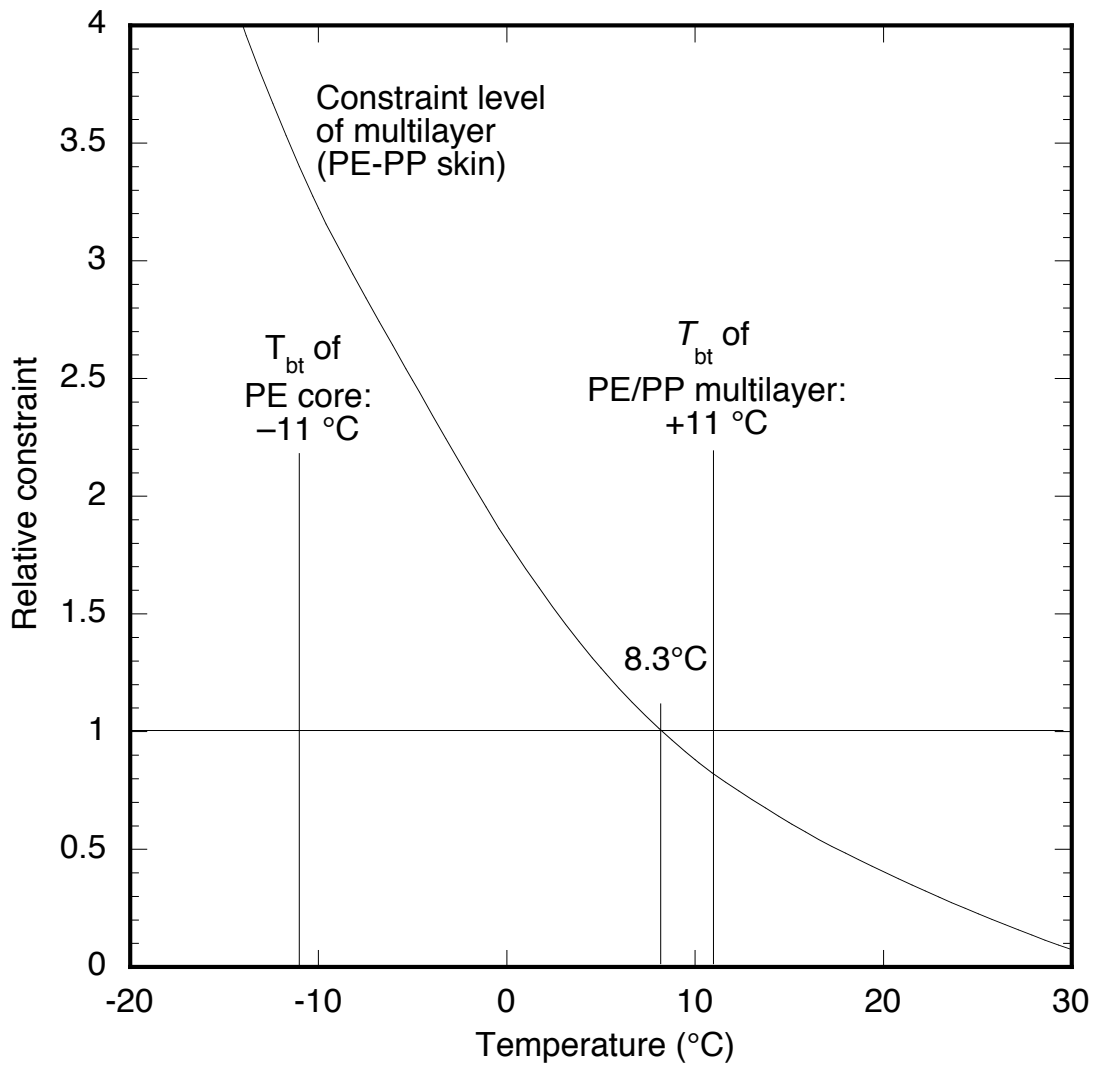




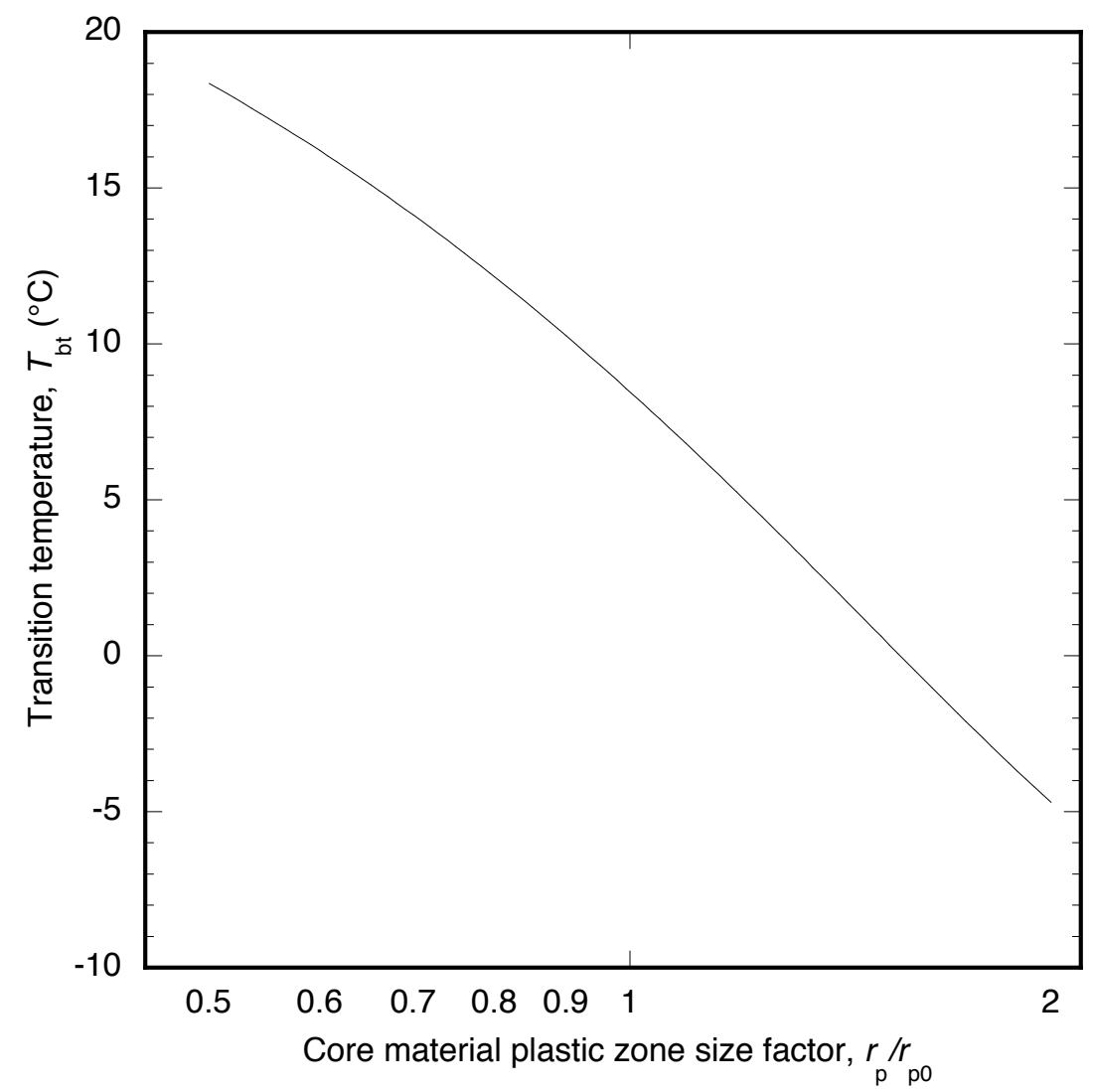




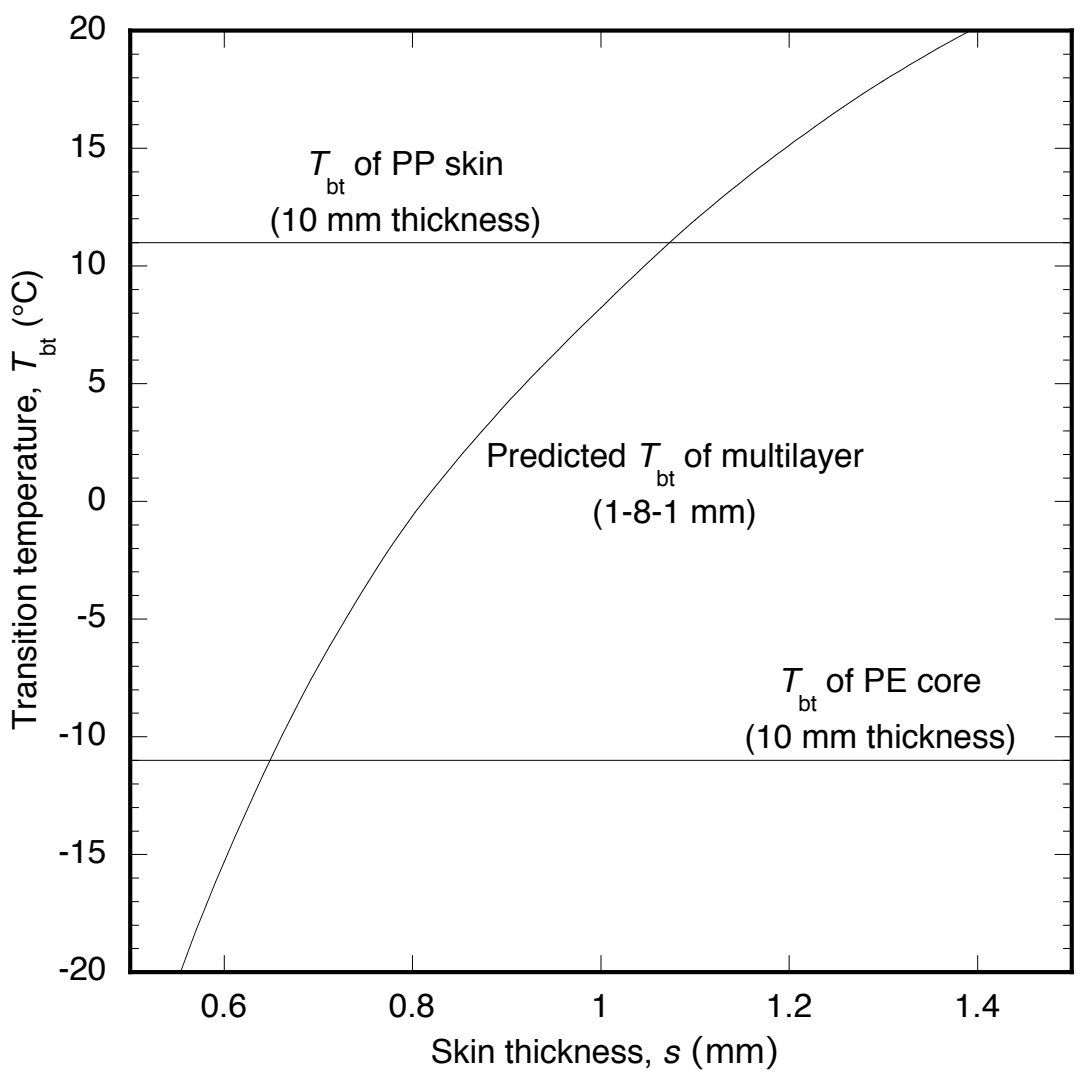

